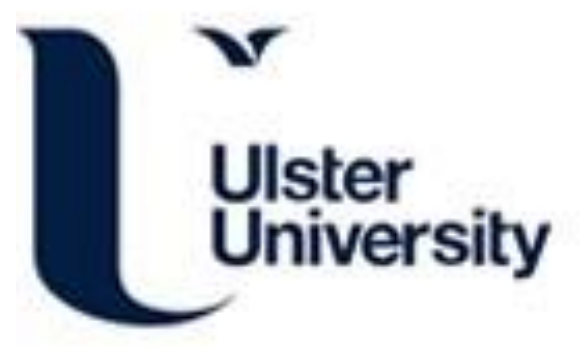

\title{
Assessing a five factor model of PTSD: Is dysphoric arousal a unique PTSD construct showing differential relationships with anxiety and depression?
}

Armour, C., Elhai, J. D., Richardson, D., Ractliffe, K., Wang, L., \& Elklit, A. (2012). Assessing a five factor model of PTSD: Is dysphoric arousal a unique PTSD construct showing differential relationships with anxiety and depression? Journal of Anxiety Disorders, 26(2), 368-376. https://doi.org/10.1016/j.janxdis.2011.12.002

Link to publication record in Ulster University Research Portal

Published in:

Journal of Anxiety Disorders

Publication Status:

Published (in print/issue): 01/01/2012

DOI:

10.1016/j.janxdis.2011.12.002

\section{Document Version}

Publisher's PDF, also known as Version of record

\section{General rights}

Copyright for the publications made accessible via Ulster University's Research Portal is retained by the author(s) and / or other copyright owners and it is a condition of accessing these publications that users recognise and abide by the legal requirements associated with these rights.

\section{Take down policy}

The Research Portal is Ulster University's institutional repository that provides access to Ulster's research outputs. Every effort has been made to ensure that content in the Research Portal does not infringe any person's rights, or applicable UK laws. If you discover content in the Research Portal that you believe breaches copyright or violates any law, please contact pure-support@ulster.ac.uk. 


\title{
Assessing a five factor model of PTSD: Is dysphoric arousal a unique PTSD construct showing differential relationships with anxiety and depression?
}

\author{
Cherie Armour ${ }^{\mathrm{a}, *}$, Jon D. Elhai ${ }^{\mathrm{b}}$, Don Richardson ${ }^{\mathrm{c}, \mathrm{d}}$, Kendra Ractliffe ${ }^{\mathrm{e}}$, Li Wang ${ }^{\mathrm{f}}$, Ask Elklit ${ }^{\mathrm{a}}$ \\ a National Centre for Psychotraumatology, University of Southern Denmark, Odense, Denmark \\ ${ }^{\mathrm{b}}$ Department of Psychology, University of Toledo, Toledo, OH, USA \\ c Operational Stress Injury Clinic, St. Joseph's Health Care London - Parkwood Hospital, University of Western Ontario, London, Ontario, Canada \\ ${ }^{\mathrm{d}}$ National Center for Operational Stress Injury-Sainte-Anne-de-Bellevue (Québec) - Veterans Affairs Canada, Canada \\ e Disaster Mental Health Institute, The University of South Dakota, Vermillion, SD, USA \\ ${ }^{\mathrm{f}}$ Institute of Psychology, Chinese Academy of Sciences, Beijing, China
}

\section{A R T I C L E I N F O}

\section{Article history:}

Received 20 July 2011

Received in revised form

28 November 2011

Accepted 4 December 2011

\section{Key words:}

Posttraumatic stress disorder

Confirmatory factor analysis

Depression

Anxiety, veterans, medical patients

\begin{abstract}
A B S T R A C T
Posttraumatic stress disorder's (PTSD) latent structure has been widely debated. To date, two four-factor models (Numbing and Dysphoria) have received the majority of factor analytic support. Recently, Elhai et al. (2011) proposed and supported a revised (five-factor) Dysphoric Arousal model. Data were gathered from two separate samples; War veterans and Primary Care medical patients. The three models were compared and the resultant factors of the Dysphoric Arousal model were validated against external constructs of depression and anxiety. The Dysphoric Arousal model provided significantly better fit than the Numbing and Dysphoria models across both samples. When differentiating between factors, the current results support the idea that Dysphoric Arousal can be differentiated from Anxious Arousal but not from Emotional Numbing when correlated with depression. In conclusion, the Dysphoria model may be a more parsimonious representation of PTSD's latent structure in these trauma populations despite superior fit of the Dysphoric Arousal model.
\end{abstract}

(c) 2011 Elsevier Ltd. All rights reserved.

\section{Introduction}

The fifth edition of the Diagnostic and Statistical Manual of Mental Disorders (DSM-5; American Psychological Association (APA)) is tentatively scheduled for publication in 2013. Successive revisions of the DSM are required to ensure that the growing knowledge base of mental disorders is reflected in diagnosis. This is particularly pertinent for posttraumatic stress disorder (PTSD) as PTSD's nosology has been at the core of extensive debate and controversy since its initial inclusion in the DSM in 1980 (DSM-III, 1980). In particular, an ongoing issue relates to how the 17 PTSD symptoms are operationalized. Currently, the DSM-IV's (APA, 1994) tripartite factor structure of Intrusion, Effortful Avoidance/Emotional Numbing and Arousal is rarely empirically supported (cf. Asmundson, Stapleton, \& Taylor, 2004). The implications of this are vast given that PTSD's factor structure is directly related to diagnostic algorithms, meaning that alternative factor structures can result in differences in PTSD's prevalence rates (Elhai, Ford, Ruggiero, \& Frueh, 2009; Forbes et al., 2011).

\footnotetext{
* Corresponding author at: The National Centre for Psychotraumatology, University of Southern Denmark, Campusvej 55, Odense, Denmark.

E-mail addresses: carmour@health.sdu.dk, armour.cherie@gmail.com (C. Armour).
}

In addition to existing evidence-based PTSD models, a recent five-factor alternative termed the Dysphoric Arousal model, proposed by Elhai et al. (2011), is promising, given support for this structure in female victims of domestic violence (Elhai et al., 2011), earthquake survivors, and violent riot victims (Wang et al., in press, Wang, Long, Li, \& Armour, 2011; Wang, Zhang, et al., 2011). The present study investigates the new Dysphoric Arousal model in a sample of Canadian veterans and in a sample of trauma-exposed American primary care medical patients. In addition, the present study will investigate each of the resultant factors' relationships with measures of depression and anxiety. This is in line with Elhai and Palmieri's (2011) proposal that CFA studies should validate their factors against external variables of interest, such as comorbid psychiatric disorders. The rationale behind their proposal was based on the fact that several studies test only the structure of certain PTSD measures without any attempt at assessing construct validity.

The extensive factor analytic research, ongoing since the 1990s, has concluded that PTSD is better operationalized in terms of a four-factor structure compared to the DSM-IV's tripartite structure. Indeed, two four-factor structures (King, Leskin, King, \& Weathers, 1998; Simms, Watson, \& Doebbeling, 2002) have received a wealth of empirical support. The King et al. Numbing model retains the original symptom groupings of Intrusion and Arousal of the DSM$I V$ tripartite structure (cf. Table 1). However, this conceptualization 
Table 1

Item distribution table for PTSD models.

\begin{tabular}{|c|c|c|c|c|}
\hline \multirow[t]{2}{*}{ PTSD symptoms } & \multicolumn{4}{|c|}{ PTSD models } \\
\hline & DSM-IV & King: Numbing Model & Simms: Dysphoria Model & 5-Factor: Dysphoric Arousal Model \\
\hline B1: Intrusive thoughts & I & I & I & I \\
\hline B2: Nightmares & I & I & I & I \\
\hline B3: Reliving trauma & I & I & I & I \\
\hline B4: Emotional cue reactivity & I & I & I & I \\
\hline B5: Physiological cue reactivity & I & I & I & I \\
\hline $\mathrm{C} 1$ : Avoidance of thoughts & $\mathrm{AV} / \mathrm{N}$ & $\mathrm{AV}$ & AV & AV \\
\hline C2: Avoidance of reminders & $\mathrm{AV} / \mathrm{N}$ & AV & AV & AV \\
\hline C3: Trauma-related amnesia & $\mathrm{AV} / \mathrm{N}$ & $\mathrm{N}$ & $\mathrm{D}$ & $\mathrm{N}$ \\
\hline C4: Loss of interest & $\mathrm{AV} / \mathrm{N}$ & $\mathrm{N}$ & $\mathrm{D}$ & $\mathrm{N}$ \\
\hline C5: Feeling detached & $\mathrm{AV} / \mathrm{N}$ & $\mathrm{N}$ & $\mathrm{D}$ & $\mathrm{N}$ \\
\hline C6: Feeling numb & $\mathrm{AV} / \mathrm{N}$ & $\mathrm{N}$ & $\mathrm{D}$ & $\mathrm{N}$ \\
\hline C7: Hopelessness & $\mathrm{AV} / \mathrm{N}$ & $\mathrm{N}$ & $\mathrm{D}$ & $\mathrm{N}$ \\
\hline D1: Sleeping Difficulties & A & A & $\mathrm{D}$ & DA \\
\hline D2: Irritability & A & A & $\mathrm{D}$ & $\mathrm{DA}$ \\
\hline D3: Concentration Difficulties & A & A & $\mathrm{D}$ & $\mathrm{DA}$ \\
\hline D4: Overly alert & A & A & A & $\mathrm{AA}$ \\
\hline D5: Exaggerated Startle Response & A & A & A & $\mathrm{AA}$ \\
\hline
\end{tabular}

Note: $\mathrm{R}=$ Intrusion; $\mathrm{AV}=$ Avoidance; $\mathrm{N}=$ Numbing; $\mathrm{A}=$ Arousal; $\mathrm{D}=$ Dysphoria; $\mathrm{DA}=$ Dysphoric Arousal; $\mathrm{AA}=\mathrm{Anxious}$ Arousal.

differs in that the Avoidance/Numbing factor is split into two distinct factors of Avoidance and Numbing. Support for separating the Avoidance/Numbing factor of the DSM-IV model is reviewed in Asmundson et al. (2004). For example, Asmundson et al. (2004) discussed how the mechanisms underlying Numbing and Avoidance differ in that Numbing serves as an automatic response to Arousal symptomatology whereas Avoidance serves as a deliberate means of escaping trauma-related stimuli (Foa et al., 1992). The authors also discussed that given these differing underlying mechanisms, each factor may require and respond to different treatment approaches. Empirical support for the Numbing model is strong (most recently in Armour, Elhai, et al., 2011, Armour, Layne, et al., 2011; Elhai, Palmieri, Biehn, Frueh, \& Magruder, 2010; Grubaugh, Long, Elhai, Frueh, \& Magruder, 2010; Hoyt \& Yeater, 2010; Mansfield, Williams, Hourani, \& Babeu, 2010).

Simms et al. (2002) modified the Numbing model, to create a new four-factor model, by employing a two-stage process. First, they separated three of the Arousal symptoms (D1 = sleeping difficulties, D2 = irritability, D3 = concentration difficulties) from the Arousal factor. Second, they added the three Arousal symptoms to the Numbing symptoms to create a new Dysphoria factor (cf. Table 1). The Simms et al. Dysphoria model was based on the proposal that a number of PTSD's symptoms reflected a general distress component and that these symptoms, i.e., the Dysphoria factor items, were related to and overlap with other mood and anxiety disorders. Again, support for the Dysphoria model is strong (most recently in Armour, McBride, Shevlin, \& Adamson, 2011c; Armour \& Shevlin, 2010; Carragher, Mills, Slade, Teesson, \& Silove, 2010; Engdahl, Elhai, Richardson, \& Frueh, 2011; Naifeh, Richardson, Del Ben, \& Elhai, 2010; Pietrzak, Goldstein, Malley, Rivers, \& Southwick, 2010).

Notably, the Numbing and Dysphoria models differ only on their placement of three items (D1-D3) and are generally selected as superior on the basis of minimal differences in fit indices (cf. Armour \& Shevlin, 2010). Since both models receive support in a variety of samples with similar charecteristics, there is no clear consensus as to which of the two models provides superior fit. This lack of concensus holds despite a recent meta-analytic study of 40 PTSD studies, which showed mild support for the Dysphoria model over the Numbing model (Yufik \& Simms, 2010) and multiple efforts to elucidate model superiority based on differences in assessment and measurement related conditions (Armour, Elhai, et al., 2011; Armour, Layne, et al., 2011; Elhai et al., 2009; Palmieri, Weathers, Difede, \& King, 2007).
Combined these results suggest that both four-factor models represent the latent structure of PTSD well but that superiority is dependant on certain sample charecteristics. In other words, one model is not necessarily always going to be superior to the other irrespective of the number of CFA studies conducted. Recently, the appropriateness of these two models, with regard to model superiority, has been called into question with the proposal of a new five-factor Dysphoric Arousal model (Elhai et al., 2011).

Elhai et al. (2011) stated that one issue with the Dysphoria model, based on the two modifications to the Numbing model (as indicated above), is that we do not know which of the two modifications have resulted in superior fit for this model. This issue, combined with research highlighting that PTSD items D1-D3 differ conceptually and empirically from both the remaining Arousal symptoms and from the Numbing symptoms (Watson, 2005), and that items D1-D3 did not load well on Arousal or Dysphoria factors (Shevlin, McBride, Armour, \& Adamson, 2009), led Elhai et al. to propose a model which involves only the first modification made by Simms et al. (2002) to the Numbing model. Specifically, Elhai et al. proposed a fifth PTSD factor comprised of only D1, D2, and D3, termed Dysphoric Arousal (cf. Table 1). Using data from a sample of domestic violence victims $(N=252)$ Elhai et al. specified and estimated the Emotional Numbing model, the Dysphoria model, and the Dysphoric Arousal model. The Dysphoric Arousal model resulted in significantly better fit, and has since been further supported in data from Chinese earthquake victims, victims of violent riots, and Danish elderly bereaved (Armour, O'Connor, Elklit, \& Elhai, in press; Wang et al., in press; Wang, Long, et al., 2011; Wang, Zhang, et al., 2011). However, one potential limitation with the newly proposed Dysphoric Arousal model is that studies have reported high interfactor correlations between the Emotional Numbing and Dysphoric Arousal factors and the Dysphoric Arousal and Anxious Arousal factors, particularly in relation to the former (cf. Armour et al. in press; Wang, Zhang, et al., 2011). Furthermore, Armour et al. (in press) also reported that Dysphoric Arousal and Emotional Numbing factors were equally related to depression in a sample of elderly bereaved participants and are thus potentially one in the same. Limitations such as these highlight the need to judge models based on a balance of statistical fit, substantive meaning, and parsimony.

In the present study we specified and estimated the Numbing and Dysphoria models, testing them against the Dysphoric Arousal model, to further test this model in additional samples. The analyses were conducted in both a sample of Canadian veterans 
with a military trauma history and a sample of American traumaexposed primary care medical patients. Next, we assessed whether the Dysphoric Arousal factor (in contrast to Emotional Numbing and Anxious Arousal factors) was differentially related to anxiety, rumination and depression (in contrast to Emotional Numbing and Anxious Arousal factors). The rationale for doing so was based on the fact that depression and anxiety are both highly comorbid with PTSD, with anxiety fundamentally at the core of PTSD (Elhai, Grubaugh, Kashdan, \& Frueh, 2008), and rumination being highlighted as an important vulnerability factor for the development of PTSD (Elwood, Hahn, Olatunji, \& Williams, 2009). We hypothesized that the Dysphoric Arousal model would provide superior fit compared to the Emotional Numbing and Dysphoria models in both samples. We further hypothesized that the Dysphoric Arousal factor would be differentially related to measures of depression and anxiety compared to alternative factors, based on the abovementioned rationale, albeit one previous study reporting that Dysphoric Arousal and Emotional Arousal could not be differentiated based on their associations with a measure of depression (Armour et al., in press).

\section{Study 1}

\subsection{Method}

\subsubsection{Participants and procedure}

We analyzed data from a clinical dataset of 408 Canadian veterans with military trauma exposure. Participants were referred by their medical provider or disability pension officer to Veterans Affairs Canada, or to a community mental health clinic specializing in treating veterans where their evaluations were funded by Veterans Affairs Canada. Institutional Review Board approval was obtained from the Office of Research Ethics at the University of Western Ontario for use of these clinical data. All participants were evaluated for PTSD. Two subjects were removed from analyses because they were not administered the PTSD Checklist (see below), leaving an effective sample of 406 .

Participants were mostly men $(n=387 ; 96 \%)$, and ranged in age from 22 to $93(M=55.84, S D=19.6)$. Nearly half did not receive a high school diploma $(n=195 ; 48 \%)$, while the rest completed high school $(n=138 ; 34 \%)$, some college $(n=49 ; 12 \%)$, or a bachelor's degree ( $n=24 ; 6 \%)$. Regarding military history, 64\% ( $n=259)$ served as peacekeeping veterans, 29\% $(n=118)$ served in World War II, and 7\% $(n=28)$ served in the Korean War. The number of deployments ranged from 0 to $7(M=1.46, S D=1.22)$. All participants endorsed at least one direct trauma exposure item on the Life Events Checklist (see below for details), with the most commonly endorsed directly experienced traumas including war zone exposure $(n=285 ; 82 \%)$, transportation accident $(n=279 ; 79 \%)$, severe human suffering $(n=266 ; 75 \%)$, fire or explosion $(n=265 ; 74 \%)$, and assault with a weapon $(n=264 ; 74 \%)$.

\subsubsection{Measures}

Several clinician interviews and self-report instruments were administered by the clinic's mental health professionals. Instruments relevant to the present study are described below.

Demographics. Participants completed a questionnaire inquiring about the various demographic and military variables described above.

Trauma exposure. The life events checklist (LEC) (Blake et al., 1995 ) is a self-report measure assessing exposure to 16 potentially traumatic events meeting PTSD's criterion A1, using a 5-point nominal scale ( 1 = "happened to me"; 2 = "witnessed it"; 3 = "learned about it"; 4 = "not sure"; and 5 = "does not apply"). High test-retest reliability has been demonstrated (mean kappa coefficient of .61 for the direct exposure items, and .41 for the indirect exposure items) (Gray et al., 2004). Convergent validity with other trauma exposure measures and prediction of PTSD symptomatology have been revealed (Gray, Litz, Wang, \& Lombardo, 2004).

PTSD symptoms. The PTSD Checklist (PCL: Weathers et al., 1993) is a 17-item self-report PTSD instrument that maps onto DSM-IV's PTSD symptom criteria. Items are queried on a five-point Likert scale of past-month severity ( $1=$ "not at all," to $5=$ "extremely"). The PCL has demonstrated internal consistency between .90-.95, test-retest reliability approaching .90, and convergent validity with structured PTSD diagnostic interviews and other PTSD self-report measures (reviewed in McDonald \& Calhoun, 2010). For this study, we used the PCL-Military version (PCL-M), which queries PTSD symptoms specifically in relation to prior stressful military experiences, with established psychometrics in military veterans (Forbes, Creamer, \& Biddle, 2001), and demonstrated adequate internal consistency in the current study (alpha $=.91$ ).

Anxiety. The Beck Anxiety Inventory (BAI; Beck, Epstein, Brown, \& Steer, 1988) is a 21-item self-report instrument of anxiety experienced over the past week, using a four-point Likert scale. Excellent test-retest reliability, and internal consistency have been found across studies (Beck, Steer, \& Garbin, 1988; Wetherell \& Areán, 1997) (alpha $=.93$ in the present study). Moderate to strong correlations have been found with other anxiety measures (Beck, Steer, et al., 1988).

Depression. The Beck Depression Inventory (BDI, Beck, Ward, Mendelson, Mock, \& Erbaugh, 1961) is a 21-item self-report instrument measuring depression. The BDI has established reliability (coefficient alpha ranging from .81 to .86) (Beck, Epstein, et al., 1988 ) and construct validity (with clinical ratings and the Hamilton Scale) ( $r s=.60-.72$ for non-psychiatric and psychiatric patients, respectively) (Beck, Epstein, et al., 1988). Coefficient alpha in this sample was .91.

In the early days of the clinic's operation, the BDI was used ( $n=130$ ), but later the clinic adopted the BDI-II (Beck, Steer, Ball, \& Ranieri, 1996) ( $n=253)$. The BDI-II is a 21-item instrument measuring depression, modified from the original BDI for consistency with DSM-IV diagnostic criteria for major depressive disorder. One-week test-retest reliability is high $(r=.93)$ (Beck, Steer, \& Brown, 1996). Coefficient alpha has ranged in the low .90s with psychiatric outpatients (Beck, Steer, Ball, et al., 1996; Beck, Steer, \& Brown, 1996) (.93 in the present study). The BDI-II converged better with the Hamilton Psychiatric Rating Scale for Depression $(r=.71)$ than with the revised Hamilton Rating Scale for Anxiety $(r=.47)$ in outpatients (Beck, Steer, Ball, et al., 1996).

\subsubsection{Analysis}

The sample of 406 participants was used in primary analyses using the PCL; nominal amounts of missing item-level data were present (1-2 items each), which were estimated using maximum likelihood (ML) estimation procedures (Graham, 2009). In validation analyses using the BDI, BDI-II and BAI, 377 subjects were administered the BAI; as per above, 130 subjects were administered the BDI, and 253 subjects were administered the BDI-II. We used ML procedures to estimate missing values among those administered these instruments, subsequently summing their total scale scores.

All analyses were conducted using Mplus 6 software (Muthén \& Muthén, 2010, 1998-2010). CFA was specified based on the four-factor (intercorrelated) Numbing and Dysphoria models; all residual error covariances were fixed to zero. Since the PCL items were normally distributed (no skewness/kurtosis values $>1.35$ ), we used maximum likelihood estimation in CFA. We tested the Numbing model against the five-factor model (splitting the dysphoric arousal symptoms from the Numbing model's arousal factor). Additionally, we tested the Dysphoria model against the 
Table 2

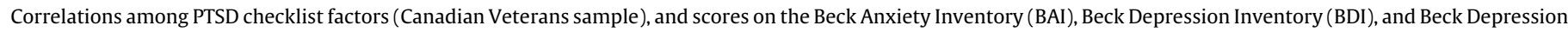
Inventory-II (BDI-II).

\begin{tabular}{|c|c|c|c|c|c|c|c|c|}
\hline & Intrusion & Avoidance & Numbing & Dysphoric Arousal & Anxious Arousal & BAI & BDI & BDI-II \\
\hline Intrusion & 1 & & & & & & & \\
\hline Avoidance & .87 & 1 & & & & & & \\
\hline Numbing & .66 & .74 & 1 & & & & & \\
\hline Dysphoric Arousal & .77 & .82 & .98 & 1 & & & & \\
\hline Anxious Arousal & .82 & .81 & .82 & .90 & 1 & & & \\
\hline BAI & $.58^{\mathrm{a}}$ & $.55^{\mathrm{a}}$ & $.64^{\mathrm{a}}$ & $.66^{\mathrm{a}}$ & $.64^{\mathrm{a}}$ & 1 & & \\
\hline BDI & $.50^{\mathrm{b}}$ & $.58^{\mathrm{b}}$ & $.75^{\mathrm{b}}$ & $.75^{\mathrm{b}}$ & $.61^{\mathrm{b}}$ & $.70^{\mathrm{d}}$ & 1 & \\
\hline BDI-II & $.59^{c}$ & $.59^{c}$ & $.78^{\mathrm{c}}$ & $.78^{c}$ & $.68^{c}$ & $.75^{c}$ & $\mathrm{~N} / \mathrm{A}$ & 1 \\
\hline
\end{tabular}

All sample sizes were 406, except as otherwise noted. N/A = Not applicable, since no subjects were administered both the BDI and BDI-II.

a $n=377$.

b $n=130$.

c $n=253$.

d $n=123$.

five-factor model (splitting the Dysphoric Arousal symptoms from the Dysphoria model's dysphoria factor). In scaling the factors, we fixed all factor variances to a value of 1 .

Goodness of fit indices are reported, including the comparative fit index (CFI), Tucker-Lewis index (TLI), root mean square error of approximation (RMSEA), and standardized root mean square residual (SRMR). Models fitting very well are indicated by CFI and $\mathrm{TLI} \geq .95$, RMSEA $\leq .06$, and SRMR $\leq .08$ (Hu \& Bentler, 1999). All tests were two-tailed. Comparing nested models by examining differences in traditional goodness of fit indices is not appropriate, and inaccurate (Fan \& Sivo, 2009). Therefore, in comparing a given four-factor model with the five-factor model, we used a chisquare difference test for nested models. We also present Bayesian Information Criterion (BIC) values for comparing the Numbing and Dysphoria models; chi-square difference testing is not possible between the four-factor models since they are not nested within one another. In comparing BIC values between models, a 10point BIC difference represents a 150:1 likelihood and "very strong" $(p<.05)$ support that the model with the smaller BIC value fits best; a difference in the 6 to 9 point range indicates "strong" support (Kass \& Raftery, 1995; Raftery, 1995).

\section{Results}

A CFA for the four-factor Numbing model did not result in an excellent fit (though would probably be regarded as an "adequate" fit), $\chi^{2}(113, N=406)=342.72, p<.001, \mathrm{CFI}=.93, \mathrm{TLI}=.91$, RMSEA $=.07$ (90\% CI: .06-.08), SRMR $=.05$, BIC $=20,925.43$. The fivefactor Dysphoric Arousal model yielded some evidence for an excellent fit, $\chi^{2}(109, N=406)=292.83, p<.001, \mathrm{CFI}=.94$, TLI $=.93$, RMSEA $=.06$ (90\% CI: .06-.07), SRMR=.05, BIC=20,899.56; and based on chi-square difference testing, the five-factor model fit significantly better than the Numbing model, $\chi_{\text {change }}^{2}(4$, $N=406)=49.89, p<.0001$.

ACFA for the four-factor Dysphoria model did not result in excellent fit (but perhaps “adequate"), $\chi^{2}(113, N=406)=314.49, p<.001$, $\mathrm{CFI}=.93, \mathrm{TLI}=.92$, RMSEA $=.07$ (90\% CI: .06-.08), SRMR $=.05$, $\mathrm{BIC}=20,897.17$. This model did not fit substantially better or worse than the Numbing model, based on BIC value comparison. The five-factor model fit significantly better than the Dysphoria model, $\chi_{\text {change }}^{2}(4, N=406)=21.66, p<.001$.

Next, we assessed whether the Dysphoric Arousal factor (in contrast to Emotional Numbing and Anxious Arousal factors) was differentially related to anxiety and depression. Factor scores generated from the five-factor Dysphoric Arousal model analysis were used in conjunction with total scores for the BAI, BDI, and BDI-II. The difference between a number of correlations was statistically significant. The Dysphoric Arousal factor was more related $(r=.66)$ than the Emotional Numbing factor $(r=.64)$ to BAI scores, $t(374$, $n=377)=3.00, p=.003$. The Dysphoric Arousal factor was more related $(r=.75)$ than the Anxious Arousal factor $(r=.61)$ to BDI scores, $t(127, n=130)=5.35, p<.0001$, and more related $(r=.78)$ than the Anxious Arousal factor $(r=.68)$ to BDI-II scores, $t(250$, $n=253)=5.63, p<.0001$. A factor intercorrelation matrix including PTSD's factors and the BAI, BDI and BDI-II can be found in Table 2.

\section{Study 2}

\subsection{Method}

\subsubsection{Participants and procedure}

Study 2 was conducted in the waiting room of a public, primary care medical clinic serving local residents in the downtown area of a medium-sized Midwestern city. The clinic is affiliated with the state university's medical school. It is a fee-for-service clinic, but also provides healthcare services at no charge to indigent persons. Data collection occurred from January to June of 2010. Adults (between ages 18 and 65) consecutively presenting for primary care medical appointments were invited by a psychology graduate student to participate in a brief, voluntary paper-and-pencil survey, without monetary compensation. Initially, 551 individuals were invited. However, 52 of these persons were excluded from participation because of an inability to speak or read English, or for not being patients of the clinic. Of 499 eligible patients, 411 agreed to participate, for a response rate of $82 \%$.

After excluding participants without trauma exposure and for substantial missing data (described below, leaving 310 subjects), participants included 113 men (36.5\%) and 197 women (63.5\%). The sample ranged in age from 19 to 64 years $(M=42.44, S D=11.63)$, and in schooling from 7 to 19 years $(M=12.89, S D=2.11)$. The majority was Caucasian ( $n=248,80.0 \%$, with additional representation primarily from Native Americans $(n=30,9.7 \%)$, Hispanics ( $n=29,9.4 \%$ ), and African Americans ( $n=15,4.8 \%$ ) (these categories are not mutually exclusive). Annual household income level was primarily less than $\$ 15 \mathrm{~K}(n=131,42.3 \%)$, between $\$ 15 \mathrm{~K}$ and $\$ 25 \mathrm{~K}$ ( $n=84,27.1 \%)$ or between $\$ 25 \mathrm{~K}$ and $\$ 35 \mathrm{~K}(n=49,15.8 \%)$.

The most prevalent traumatic events endorsed included learning of the unexpected violent or accidental death of a loved one ( $n=181,58.6 \%$ of the 310 participants with any trauma exposure), adult physical assault ( $n=167,53.9 \%$ ), child physical abuse $(n=136,43.9 \%)$, and being in a life-threatening accident $(n=131$, 42.3\%). The most prevalent event that participants nominated as the worst trauma included the unexpected violent/accidental death of a loved one $(n=98,32.0 \%)$, child physical abuse $(n=39$, $12.7 \%)$, being in a life-threatening accident $(n=34,11.1 \%)$, having a 
life-threatening illness $(n=33,10.8 \%)$, and sexual assault $(n=31$, $10.1 \%$ ). The worst trauma was reported to occur an average of 4.03 years prior $(S D=7.06)$. The majority endorsed intense fear, helplessness or horror immediately after the worst event $(n=264$, 86.0\%).

\subsubsection{Measures}

Several measures were administered as part of a larger project, with the following relevant to the present paper.

Demographics survey. A demographic questionnaire inquired about information such as age, gender, years of education, employment status, etc.

Trauma exposure. The Stressful Life Events Screening Questionnaire (SLESQ, Goodman, Corcoran, Turner, Yuan, \& Green, 1998) is a comprehensive self-report screening measure that assesses 12 DSM-IV PTSD criterion A traumatic events and a 13th "other" catchall item. Adequate test-retest reliability and convergent validity have been reported (kappa values of .73, and .64, respectively) (Goodman et al., 1998). Upon completion of the SLESQ participants were asked to nominate their most distressing traumatic event.

PTSD. The PTSD Symptom Scale-Self-Report (PSS, Foa, Riggs, Dancu, \& Rothbaum, 1993) is a 17-item DSM-IV-based self-report PTSD symptom measure. Symptom severity is measured via a fourpoint Likert scale $(0=$ "not at all," to $3=$ " $5+$ times per week/very much/almost always") for symptoms experienced over the previous two weeks. Participants were instructed to complete the PSS based upon the most distressing criterion A traumatic event reported on the SLESQ. Psychometric properties include test-retest reliability of $r=.74$, and internal consistency of .91 (Foa et al., 1993) (.94 in the present sample). Convergent validity has been demonstrated with other similar measures, with PTSD diagnostic utility found against the Structured Clinical Interview for DSM (Foa et al., 1993) and Clinician Administered PTSD Scale (Coffey, Gudmundsdottir, Beck, Palyo, \& Miller, 2006).

Anxiety. The State Trait Anxiety Inventory (STAI) (Spielberger, Gorsuch, Lushene, Vagg, \& Jacobs, 1983) queries 20 items reflecting state-dependent anxiety and 20 items reflecting trait-dependent anxiety, on a Likert-based scale of " $1=$ Not at all; or Almost never" to " $4=$ Very much so; or Almost always." Psychometric studies have demonstrated excellent internal consistency for the STAI scale and its subscales, including Cronbach's alpha ranging from .89 to .92 (Barnes, Harp, \& Jung, 2002; Spielberger et al., 1983) (.89-.94 in the current sample). Test-retest reliability is good (average $r=.88$ ) (Barnes et al., 2002). The STAI correlates .80 with the Taylor Manifest Anxiety Scale, and .75 with the Institute of Personality and Ability Test (IPAT) Anxiety Scale (Spielberger et al., 1983). Construct validity has also been supported through confirmatory factor analysis (Vigneau \& Cormier, 2008).

Rumination. The Ruminative Thought Style Questionnaire (RTS) (Brinker \& Dozois, 2009) includes 20 items of repetitive, recurrent, uncontrollable, and intrusive ruminative thoughts, with seven Likert response options ranging from " $1=$ Does not describe me at all" to "7 = Describes me very well." Adequate internal consistency has been demonstrated (Cronbach's alpha $=.95)($ Brinker \& Dozois, 2009) $(.94$ in the current sample). RTS total scores converge well against those of the Beck Depression Inventory-2; however, based on analyses controlling for situational depression, it appears that the RTS taps a global ruminative style (Brinker \& Dozois, 2009).

\subsubsection{Analysis}

4.1.3.1. Exclusions and missing data. Among the initial 411 participants, two participants failed to answer any items on the SLESQ. Among the 409 remaining participants, 329 (80\%) endorsed at least one traumatic event, corroborating trauma exposure rates from primary care settings (e.g., Elhai, Patrick, Anderson, Simons, \&
Frueh, 2006; McQuaid, Pedrelli, McCahill, \& Stein, 2001); however, 19 subjects did not answer any PSS items, leaving an effective sample size of 310 subjects.

Among the 310 participants, only six subjects were missing values on the PSS (one item each), estimated using ML procedures (Graham, 2009) via a pairwise-present approach. For validation analyses that implemented the RTS and STAI, 1-2 missing items each were evident among 12 participants on the RTS and 17 participants on the STAI. We used ML procedures to estimate missing values among those administered these instruments, subsequently scoring their total scale scores; however, we excluded from analyses participants who failed to provide any STAI $(n=1)$ or RTS responses $(n=3)$.

We present the same analytic approach to CFA as in Study 1, with only a few exceptions. First, we treated the PSS items as ordinal variables; this decision was based on substantial research demonstrating that treating ordinal data (with fewer than five response options) as continuously scaled, typically results in violations of the requirement for a linear association between factors and observed variables, biased parameter estimates that are difficult to interpret accurately, model misspecification, and failure to demonstrate true model fit (e.g., Flora \& Curran, 2004; Wirth \& Edwards, 2007). Consequently, we generated a polychoric (rather than Pearson) covariance matrix, and probit regression coefficients in the CFAs. We therefore implemented robust weighted least squares estimation with a mean- and variance-adjusted chi-square (WLSMV) for the CFAs, the preferred estimation method for ordinal items (Flora \& Curran, 2004; Wirth \& Edwards, 2007). Second, chi-square difference tests comparing a given four-factor model with the five-factor model implemented a correction factor, given the non-normally distributed WLSMV chi-square value (Muthén \& Muthén, 2006). Third, BIC values are only computable using an ML estimator; therefore, since we used the WLSMV estimator, we did not report BIC values. Fourth, we do not report the SRMR fit statistic, since it is not sensitive for ordinal items ( $\mathrm{Yu}$, 2002).

\section{Results}

A CFA for the four-factor Numbing model provided some evidence for an excellent fit, robust $\chi^{2}(113, N=310)=325.61, p<.001$, $\mathrm{CFI}=.98, \mathrm{TLI}=.97, \mathrm{RMSEA}=.08(90 \% \mathrm{CI}: .07-.09)$. The five-factor Dysphoric Arousal model also yielded evidence for an excellent fit, robust $\chi^{2}(109, N=310)=314.39, p<.001, C F I=.98, T L I=.97$, RMSEA $=.08$ (90\% CI: .07-.09); and based on chi-square difference testing, the Dysphoric Arousal model fit significantly better than the Numbing model, $\chi_{\text {change }}^{2}(4, N=310)=17.73, p=.001$.

A CFA for the four-factor Dysphoria model provided some evidence for an excellent fit, robust $\chi^{2}(113, N=310)=347.14, p<.001$, $\mathrm{CFI}=.97, \mathrm{TLI}=.97, \mathrm{RMSEA}=.08$ (90\% CI: .07-.09). The five-factor model fit significantly better than the Dysphoria model, $\chi_{\text {change }}^{2}(4$, $N=310)=29.95, p<.001$.

Next, we assessed whether the Dysphoric Arousal factor (in contrast to Emotional Numbing and Anxious Arousal factors) was differentially related to anxiety and rumination. Factor scores generated from the five-factor model analysis were used in conjunction with total scores for the STAI's trait anxiety and state anxiety scales, and RTS. The only difference in correlations that approached statistical significance was that the Dysphoric Arousal factor was slightly (but not significantly) more related $(r=.31)$ than the Anxious Arousal factor $(r=.28)$ to state anxiety scores, $t(304, n=307)=1.83$, $p=.07$. A factor intercorrelation matrix including PTSD's factors and the STAI's trait and state anxiety scales, and RTS, can be found in Table 3. The standardized factor loading for each model, across both samples, can be found in Table 4 . 
Table 3

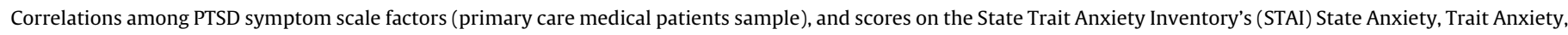
and Ruminative Thought Style Questionnaire (RTS).

\begin{tabular}{|c|c|c|c|c|c|c|c|c|}
\hline & Intrusion & Avoidance & Numbing & Dysphoric Arousal & Anxious Arousal & State Anxiety & Trait Anxiety & Rumination \\
\hline Intrusion & 1 & & & & & & & \\
\hline Avoidance & .96 & 1 & & & & & & \\
\hline Numbing & .95 & .98 & 1 & & & & & \\
\hline Dysphoric Arousal & .90 & .95 & .94 & 1 & & & & \\
\hline Anxious Arousal & .83 & .94 & .93 & .97 & 1 & & & \\
\hline State Anxiety & $.33^{\mathrm{a}}$ & $.31^{\mathrm{a}}$ & $.31^{\mathrm{a}}$ & $.31^{\mathrm{a}}$ & $.28^{\mathrm{a}}$ & 1 & & \\
\hline Trait Anxiety & $.40^{\mathrm{a}}$ & $.39^{\mathrm{a}}$ & $.40^{\mathrm{a}}$ & $.39^{\mathrm{a}}$ & $.37^{\mathrm{a}}$ & $.76^{\mathrm{a}}$ & 1 & \\
\hline Rumination & .36 & .38 & .38 & .38 & .38 & $.41^{\mathrm{a}}$ & $.60^{\mathrm{a}}$ & 1 \\
\hline
\end{tabular}

All sample sizes were 310 except as otherwise noted.

a $n=307$

Table 4

Stardardized factor loadings for the Emotional Numbing, Dysphoria and Dysphoric Arousal Models.

\begin{tabular}{|c|c|c|c|c|c|c|}
\hline \multirow[t]{3}{*}{ PTSD items } & \multicolumn{6}{|c|}{ Standardized factor loadings } \\
\hline & \multicolumn{3}{|l|}{ Study 1} & \multicolumn{3}{|l|}{ Study 2} \\
\hline & $\begin{array}{l}\text { King: Numbing } \\
\text { Model }\end{array}$ & $\begin{array}{l}\text { Simms: Dysphoria } \\
\text { Model }\end{array}$ & $\begin{array}{l}\text { 5-Factor: } \\
\text { Dysphoric Arousal } \\
\text { Model }\end{array}$ & $\begin{array}{l}\text { King: Numbing } \\
\text { Model }\end{array}$ & $\begin{array}{l}\text { Simms: Dysphoria } \\
\text { Model }\end{array}$ & $\begin{array}{l}\text { 5-Factor: Dysphoric } \\
\text { Arousal Model }\end{array}$ \\
\hline 1. Intrusive thoughts & .78 & .77 & .77 & .86 & .86 & .86 \\
\hline 2. Nightmares & .72 & .71 & .71 & .85 & .85 & .85 \\
\hline 3. Reliving trauma & .75 & .76 & .76 & .78 & .78 & .78 \\
\hline 4. Emotional cued reactivity & .71 & .71 & .71 & .90 & .91 & .91 \\
\hline 5. Physiological cued reactivity & .75 & .75 & .75 & .91 & .91 & .91 \\
\hline 6. Avoidance of thoughts & .71 & .71 & .71 & .87 & .87 & .87 \\
\hline 7. Avoidance of reminders & .81 & .81 & .81 & .87 & .87 & .87 \\
\hline 8. Trauma-related amnesia & .31 & .32 & .31 & .75 & .74 & .75 \\
\hline 9. Loss of interest & .71 & .71 & .72 & .57 & .56 & .57 \\
\hline 10. Feeling detached & .84 & .81 & .84 & .88 & .86 & .88 \\
\hline 11. Feeling numb & .81 & .79 & .81 & .89 & .88 & .89 \\
\hline 12. Hopelessness & .62 & .63 & .62 & .85 & .83 & .85 \\
\hline 13. Difficulty sleeping & .58 & .56 & .58 & .85 & .82 & .86 \\
\hline 14. Irritable/angry & .67 & .68 & .69 & .85 & .82 & .87 \\
\hline 15. Difficulty concentrating & .69 & .70 & .71 & .86 & .83 & .87 \\
\hline 16. Overly alert & .70 & .79 & .78 & .88 & .91 & .91 \\
\hline 17. Easily startled & .68 & .77 & .78 & .84 & .88 & .88 \\
\hline
\end{tabular}

\section{Discussion}

In the present study we specified and estimated the four-factor Numbing and Dysphoria models and the newly proposed Dysphoric Arousal model across two samples - Canadian veterans and Primary Care medical patients. The Dysphoric Arousal model provided significantly better fit, based on chi-square difference testing, than the Emotional Numbing model and the Dysphoria model across both samples, supporting results from other recent studies of domestic violence victims (Elhai et al., 2011), Chinese earthquake victims and Chinese victims of violent riots (Wang et al., in press; Wang, Long, et al., 2011; Wang, Zhang, et al., 2011). However, it is important to highight that the Dysphoric Arousal model provided only minimually superior fit in comparison to the Numbing and Dysphoria models. Notably, selecting a model as superior on the basis of minimal differences of fit is not uncommon in the factor analytic literature (reviewed in Armour \& Shevlin, 2010). Indeed, the Dysphoric Arousal and Numbing models provided identical fit on the majority of fit indices (CFI, TLI, RMSEA) within the Canadian Veterans sample. In addition, the Dysphoric Arousal factor of the Dysphoric Arousal model correlated highly with the Numbing factor (.98) in the Canadian Veteran sample and with the Anxious Arousal factor (.97) in the Primary Care patients sample. The strength of these correlations exceeds those usually found for the intercorrelations of the four-factor Numbing and Dysphoria models and may suggest multicollinearity despite the model providing superior fit on the basis of chi-square difference testing. Indeed, some previous studies supporting the Dysphoric Arousal model have also reported high inter-factor correlations. Most notably, Wang, Zhang, et al. (2011) reported high interfactor correlations between the Dysphoric Arousal factor and the Numbing factor across two samples (earthquake $=.85$ and violent riot $=.93$ ). The interfactor correlations were also high between the Dysphoric Arousal factor and the Anxious Arousal factor (earthquake $=.91$ and violent riot $=.82$ ). Therefore, albeit statistical support for this model appears to be growing, the model may have limitations in certain populations such as riot victims, earthquake survivors, Veterans, and Primary Care patients, suggesting that the exisiting four-factor models may be less problematic and more parsimonious in these and potentially alternative trauma populations. Despite these concerns the current study provides statistical support for the Dysphoric Arousal model over existing four-factor structures. Indeed, Elhai and Palmieri (2011) and Fan and Sivo (2009) both proposed that model selection should be based on statistical comparisons rather than simple comparisons of fit indices. Thus, we proceded to validate the five factors of the Dysphoric Arousal model against alternative measures of depression and anxiety.

Importantly, the current study is one of the first to validate the five factors of the Dysphoric Arousal model against external constructs (cf. Armour et al., in press; Wang, Zhang, et al., 2011). Indeed, as the Dysphoric Arousal model was proposed on the basis of literature suggesting that items D1-D3 differ from both the remaining Arousal symptoms and from the Numbing symptoms (Watson, 2005) it is important to differentiate the new Dysphoric Arousal factor from the Numbing factor (which is essentially the splitting of the Dysphoria factor in the Dysphoria model) and 
from the Anxious Arousal factor (which is essentially the splitting of the full Arousal factor in the DSM-IV and King models). If, for example, the Dysphoric Arousal factor cannot be differentiated from the Emotional Numbing factor on external measures of psychopathology, combined with high interfactor correlations, then albeit the Dysphoric Arousal model appears superior on chisquare differnce testing, perhaps the Dysphoria model would better reflect PTSD's underlying dimensionality. Indeed, Armour et al. (in press) reported that the Emotional Numbing and Dysphoric Arousal factors were equally related to depression in elderly bereaved participants. However, the Dysphoric Arousal factor was more related to depression compared to the Anxious Arousal factor.

The current findings concluded that in the Canadian veterans sample (Study 1), the Dysphoric Arousal factor was related to Anxiety to a greater degree than the Emotional Numbing factor. However, the correlation coefficients were almost identical (.64 vs. .66), albeit statistically significantly different. In addition, the Dysphoric Arousal factor was more related to depression, as measured by the BDI and the BDI-II compared to the Anxious Arousal factor, however these differences were small (BDI .14 and BDI-II .10). Notably, the correlations between the Emotional Numbing factor and the BDI and the BDI-II were identical to those reported between the Dysphoric Arousal factor and the BDI and the BDIII, suggesting that the Emotional Numbing and Dysphoric Arousal factors are not meaningfully differentially associated with regards to depression. In the Primary Care medical patients sample (Study 2 ), the only difference in correlations that approached statistical significance was that Dysphoric Arousal was slightly (but not significantly) more related $(r=.31)$ than Anxious Arousal $(r=.28)$ to state anxiety scores, $t(304, n=307)=1.83, p=.07$. However, given the small non-significant difference of .03 this does not warrant any further interpretation. Unfortunately, there was no depression measure included in Study 2. Therefore, the current results suggest that the Dysphoric Arousal factor shares more in common with the Emotional Numbing factor when it comes to predicting depression than it shares with the Anxious Arousal factor in Veteran samples. Unfortunately, we cannot report whether this finding is replicated in the Primary Care sample due to the absence of a depression measure.

Interestingly, although there was a statistically significant difference in the correlation coefficients between the Dysphoric Arousal factor and the Emotional Numbing factor with Anxiety, the difference in correlation coefficients is so weak that we cannot make any firm conclusions that these two factors can be differentiated by their associations with anxiety. Further to this, there were no statistical differences between these factors and state and trait anxiety in the Primary Care patient's sample. Likewise, the findings across both studies revealed that the Dysphoric Arousal and Anxious Arousal factors cannot be differentiated by their association with measures of anxiety. Thus, despite the Dysphoric Arousal model providing statistically superior fit, based on chi-square difference tests, these results may suggest that the Dysphoria model originally proposed by Simms et al. may be a more parsimonious representation of PTSD's latent structure, in these trauma populations. This is attributable to the Dysphoric Arousal factor and the Emotional Numbing factor correlating at .98 and .94 in the Veteran and Primary Care patients samples respectively. In addition, the Dysphoric Arousal and Emotional Numbing factors both provided identical associations with external measure of psychopathology, i.e., depression in the current study and as reported by Armour, Elhai, et al. (2011), Armour, Layne, et al. (2011), Armour, McBride, et al. (2011).

On a related topic, as both the Dysphoric Arousal and Emotional Numbing factors (which is essentially the Dysphoria factor of the Simms et al. Dysphoria model) are both highly associated with measures of depression $(\mathrm{BDI}=.75$ and $\mathrm{BDI}-\mathrm{II}=.78)$ these results lend support to previous suggestions of the non-specific nature of these PTSD items (Armour \& Shevlin, 2010; Armour, Elhai, et al., 2011; Armour, Layne, et al., 2011; Armour, McBride, et al., 2011; Elklit, Armour, \& Shevlin, 2010) albeit alternative reports that these items are no more related to distress or depression than other PTSD items (Armour \& Shevlin, in press; Marshall, Schell, \& Miles, 2010; Miller et al., 2010).

To date, the Dysphoric Arousal model has been shown to better represent PTSD's latent structure in various trauma populations. However, although the Dysphoric Arousal model has been statistically supported in the current study, employing Veteran and Primary Care patients samples, some may argue that the support is not particularly strong. Future research should endevour to investigate whether support for this model will or will not be found in additional trauma-exposed samples. The current study was the first to address the fit of the Dysphoric Arousal model within a heterogeneous trauma sample and so counteracts limitations of previous research which focuses on single trauma events (a limitation of CFA studies highlighted by Elhai, Gray, Docherty, Kashdan, \& Kose, 2007).

This study is not without limitations. First, this study employed the use of self-report measures across both samples. Indeed, all studies supporting the newly proposed Dysphoric Arousal model have been based on the data from self report measures, namely the PSS (Foa et al., 1993) and the PCL (Weathers et al., 1993). Notably, factor analytic research employing the use of self-report measures is common (e.g., Elklit \& Shevlin, 2007; Elklit et al., 2010; McDonald et al., 2008). However, future research needs to address whether these findings would replicate across a variety of alternative selfreport measures and clinical interviews. Second, we were unable to assess the relationships between the factors of the Dysphoric Arousal model and depression in the second study as a depression measure was not included in the original data collection. In addition, it is unclear whether the results of study one would generalize to female veterans given that study one consisted of mostly male veterans ( $n=387 ; 96 \%)$.

In conclusion, the current findings are timely with the upcoming release of the DSM-5. Notably, the proposal of a new and superior model of PTSD's latent structure would be welcomed given the ambiguity in which of the two four factor models; Numbing and Dysphoria, provides a superior representation of PTSD's latent structure. Unfortunately, the current results question whether the Dysphoric Arousal model is indeed truly superior to existing empirically supported models. Importantly, the current findings have a number of implications, i.e., they illustrate that it is imperative that any such model represents the latent structure of PTSD across all trauma populations. Furthermore, although it is true that we are looking for superior model fit, models must also be judged on the basis of parsimony and substantive meaning. Thus, whether or not, the newly proposed Dysphoric Arousal model is, on balance, superior to the existing four-factor models remains to be seen. At present, it would appear that, although the Dysphoric Arousal model is statistically superior, given high interfactor correlations, particularly between the Emotional Numbing and Dysphoric Arousal factors, combined with a notable lack of differential associations between these factors with external measures of psychopathology, perhaps, on balance the Dysphoria model remains a superior representation of PTSD's dimensionality.

\section{References}

American Psychiatric Association. (1994). Diagnostic and statistical manual of mental disorders (4th ed.). Washington, DC: American Psychiatric Association.

Armour, C., Elhai, J. D., Layne, C., Shevlin, M., Duraković-Belko, E., Djapo, N., et al. (2011). Gender differences in the factor structure of posttraumatic stress disorder symptoms in war-exposed adolescents. Journal of Anxiety Disorders, 25 , 604-611. 
Armour, C., Layne, C. M., Naifeh, J. A., Shevlin, M., Duraković-Belko, E., \& Djapo, N. (2011). Assessing the factor structure of posttraumatic stress disorder symptoms in participants with and without criterion A2 endorsement. Journal of Anxiety Disorders, 25, 80-87.

Armour, C., McBride, O., Shevlin, M., \& Adamson, G. (2011). Testing the robustness of the Dysphoria factor of the Simms et al. (2002) model of PTSD. Psychological Trauma: Theory, Research, Practice and Policy, 3, 139-147.

Armour, C., O'Connor, M., Elklit, A., \& Elhai, J. D. Assessing PTSD's latent structure in elderly bereaved European trauma victims: evidence for a five factor Dysphoric and Anxious Arousal Model. Journal of Nervous an Mental Disease, in press.

Armour, C., \& Shevlin, M. (2010). Testing the dimensionality of PTSD and the specificity of the dysphoria factor. Journal of Loss and Trauma, 15, 11-27.

Armour, C., \& Shevlin, M. Assessing the specificity of PTSD's Dysphoric items within Simms et al.'s latent structure. Journal of Nervous and Mental Disease, in press.

Asmundson, G. J. G., Stapleton, J. A., \& Taylor, S. (2004). Are avoidance and numbing distinct PTSD symptom clusters? Journal of Traumatic Stress, 17, 467-475.

Barnes, L. L. B., Harp, D., \& Jung, W. S. (2002). Reliability generalization of scores on the Spielberger state-trait anxiety inventory. Educational \& Psychological Measurement, 62, 603-618.

Beck, A. T., Epstein, N., Brown, G., \& Steer, R. A. (1988). An inventory for measuring clinical anxiety: Psychometric properties. Journal of Consulting and Clinical Psychology, 56, 893-897.

Beck, A. T., Steer, R. A., Ball, R., \& Ranieri, W. F. (1996). Comparison of beck depression inventories-IA and -II in psychiatric outpatients. Journal of Personality Assessment, 67, 588-597.

Beck, A. T., Steer, R. A., \& Brown, G. K. (1996). Manual for the beck depression inventoryII. San Antonio, TX: Psychological Corporation.

Beck, A. T., Steer, R. A., \& Garbin, M. G. (1988). Psychometric properties of the beck depression inventory: Twenty-five years of evaluation. Clinical Psychology Review, 8, 77-100.

Beck, A. T., Ward, C. H., Mendelson, M., Mock, J., \& Erbaugh, J. (1961). An inventory for measuring depression. Archives of General Psychiatry, 4, 561-571.

Blake, D. D., Weathers, F. W., Nagy, L. M., Kaloupek, D. G., Gusman, F. D., Charney, D. S., et al. (1995). The development of a clinician-administered PTSD scale. Journa of Traumatic Stress, 8, 75-90.

Brinker, J. K., \& Dozois, D. J. A. (2009). Ruminative thought style and depressed mood. Journal of Clinical Psychology, 65, 1-19.

Carragher, N., Mills, K., Slade, T., Teesson, M., \& Silove, D. (2010). Factor structure of posttraumatic stress disorder symptoms in the Australian general population. Journal of Anxiety Disorders, 24, 520-527.

Coffey, S. F., Gudmundsdottir, B., Beck, J. G., Palyo, S. A., \& Miller, L. (2006). Screening for PTSD in motor vehicle accident survivors using the PSS-SR and IES. Journal of Traumatic Stress, 19, 119-128.

Elhai, J. D., Biehn, T. L., Armour, C., Klopper, J. J., Frueh, B. C., \& Palmieri, P. A. (2011). Evidence for a unique PTSD construct represented by PTSD's D1-D3 symptoms. Journal of Anxiety Disorders, 25, 340-345.

Elhai, J. D., Ford, J. D., Ruggiero, K. J., \& Frueh, B. C. (2009). Diagnostic alterations for posttraumatic stress disorder: Examining data from the National Comorbidity Survey Replication and National Survey of Adolescents. Psychological Medicine, 39, 1957-1966.

Elhai, J. D., Gray, M. J., Docherty, A. R., Kashdan, T. B., \& Kose, S. (2007). Structural validity of the posttraumatic stress disorder checklist among college students with a trauma history. Journal of Interpersonal Violence, 22, 1471-1478.

Elhai, J. D., Grubaugh, A. L., Kashdan, T. B., \& Frueh, B. C. (2008). Empirical examination of a proposed refinement to DSM-IV posttraumatic stress disorder symptom criteria using the National Comorbidity Survey Replication data. Journal of Clinical Psychiatry, 69, 597-602.

Elhai, J. D., \& Palmieri, P. A. (2011). Posttraumatic stress disorder's factor structure: An update on the current literature and advancing a research agenda. Journal of Anxiety Disorders, 25, 849-854.

Elhai, J. D., Palmieri, P. A., Biehn, T. L., Frueh, B. C., \& Magruder, K. M. (2010). Posttraumatic stress disorder's frequency and intensity ratings are associated with factor structure differences in military veterans. Psychological Assessment, 22, $723-728$

Elhai, J. D., Patrick, S. L., Anderson, S., Simons, J. S., \& Frueh, B. C. (2006). Gender- and trauma-related predictors of use of mental health treatment services among primary care patients. Psychiatric Services, 57, 1505-1509.

Elklit, A., Armour, C., \& Shevlin, M. (2010). Testing alternative factor models of PTSD and the robustness of the dysphoria factor. Journal of Anxiety Disorders, $24,147-154$.

Elklit, A., \& Shevlin, M. (2007). The structure of PTSD symptoms: A test of alternative models using confirmatory factor analysis. British Journal of Clinical Psychology, $46,299-313$.

Elwood, L. S., Hahn, K. S., Olatunji, B. O., \& Williams, N. L. (2009). Cognitive vulnerabilities to the development of PTSD: A review of four vulnerabilities and the proposal of an integrative vulnerability model. Clinical Psychology Review, 29, 87-100.

Engdahl, R. M., Elhai, J. D., Richardson, J. D., \& Frueh, B. C. (2011). Comparing posttraumatic stress disorder's symptom structure between deployed and non-deployed veterans. Psychological Assessment, 23, 1-6.

Fan, X., \& Sivo, S. A. (2009). Using $\Delta$ goodness-of-fit indexes in assessing mean structure invariance. Structural Equation Modeling, 16, 54-67. doi:10.1080/10705510802561311

Foa, E. B., Zinbarg, R., \& Rothbaum, B. O. (1992). Uncontrollability and unpredictability in post-traumatic stress disorder: an animal model. Psychological Bulletin, $112,218-238$.
Forbes, D., Fletcher, S., Lockwood, E., O’Donnell, M., Creamer, M., Bryant, R. A., et al. (2011). Requiring both avoidance and emotional numbing in DSM-V PTSD: Will it help? Journal of Affective Disorders, 130, 483-486.

Flora, D. B., \& Curran, P. J. (2004). An empirical evaluation of alternative methods of estimation for confirmatory factor analysis with ordinal data. Psychological Methods, 9, 466-491.

Foa, E. B., Riggs, D. S., Dancu, C. V., \& Rothbaum, B. O. (1993). Reliability and validity of a brief instrument for assessing post-traumatic stress disorder. Journal of Traumatic Stress, 6, 459-473.

Forbes, D., Creamer, M., \& Biddle, D. (2001). The validity of the PTSD checklist as a measure of symptomatic change in combat-related PTSD. Behaviour Research and Therapy, 39, 977-986.

Goodman, L., Corcoran, C., Turner, K., Yuan, N., \& Green, B. L. (1998). Assessing traumatic event exposure: General issues and preliminary findings for the Stressful Life Events Screening Questionnaire. Journal of Traumatic Stress, 11, 521-542.

Graham, J. W. (2009). Missing data analysis: Making it work in the real world. Annual Review of Psychology, 60, 549-576.

Gray, M. J., Litz, B. T., Wang, J., \& Lombardo, T. W. (2004). Psychometric properties of the life events checklist. Assessment, 11, 330-341.

Grubaugh, A. L., Long, M. E., Elhai, J. D., Frueh, B. C., \& Magruder, K. M. (2010). An examination of the construct validity of posttraumatic stress disorder with veterans using a revised criterion set. Behaviour Research and Therapy, 48, 909-914.

Hoyt, T., \& Yeater, E. A. (2010). Comparison of posttraumatic stress disorder symptom structure models in Hispanic and White college students. Psychological Trauma: Theory, Research, Practice \& Policy, 2, 19-30.

Hu, L., \& Bentler, P. M. (1999). Cutoff criteria for fit indexes in covariance structure analysis: Conventional criteria versus new alternatives. Structural Equation Modeling, 6, 1-55.

Kass, R. E., \& Raftery, A. E. (1995). Bayes factors. Journal of the American Statistical Association, 90, 773-795.

King, D. W., Leskin, G. A., King, L. A., \& Weathers, F. W. (1998). Confirmatory factor analysis of the clinician-administered PTSD scale: Evidence for the dimensionality of posttraumatic stress disorder. Psychological Assessment, 10, 90-96.

Mansfield, A. J., Williams, J., Hourani, L. L., \& Babeu, L. A. (2010). Measurement invariance of posttraumatic stress disorder symptoms among U.S. military personnel. Journal of Traumatic Stress, 23, 91-99.

Marshall, G. N., Schell, T. L., \& Miles, J. N. V.(2010). All PTSD symptoms are highly associated with general distress: Ramifications for the dysphoria symptom cluster. Journal of Abnormal Psychology, 119, 126-135.

McDonald, S. D., Beckham, J. C., Morey, R., Marx, C., Tupler, L. A., \& Calhoun, P. S. (2008). Factorial invariance of posttraumatic stress disorder symptoms across three veteran samples. Journal of Traumatic Stress, 21, 309-317.

McDonald, S. D., \& Calhoun, P. S. (2010). The diagnostic accuracy of the PTSD checklist: a critical review. Clinical Psychology Review, 30, 976-987.

McQuaid, J. R., Pedrelli, P., McCahill, M. E., \& Stein, M. B. (2001). Reported trauma, post-traumatic stress disorder and major depression among primary care patients. Psychological Medicine, 31, 1249-1257.

Miller, M. W., Wolf, E. J., Harrington, K. M., Brown, T. A., Kaloupek, D. G., \& Keane, T. M. (2010). An evaluation of competing models for the structure of PTSD symptoms using external measures of comorbidity. Journal of Traumatic Stress, 23, 631-638.

Muthén, B. O., \& Muthén, L. (2006). Chi-square difference testing using the SatorraBentler scaled chi-square. Retrieved from: http://statmodel.com/chidiff.shtml

Muthén, B. O., \& Muthén, L. K. (2010). Mplus (Version 6). Los Angeles, CA.

Muthén, L. K., \& Muthén, B. O. (1998-2010). Mplus user's guide (6th ed.). Los Angeles, CA: Muthén \& Muthén.

Naifeh, J. A., Richardson, J. D., Del Ben, K. S., \& Elhai, J. D. (2010). Heterogeneity in the latent structure of PTSD symptoms among Canadian veterans. Psychological Assessment, 22, 666-674.

Palmieri, P. A., Weathers, F. W., Difede, J., \& King, D. W. (2007). Confirmatory factor analysis of the PTSD checklist and the clinician-administered PTSD scale in disaster workers exposed to the World Trade Center Ground Zero. Journal of Abnormal Psychology, 116, 329-341.

Pietrzak, R. H., Goldstein, M. B., Malley, J. C., Rivers, A. J., \& Southwick, S. M. (2010) Structure of posttraumatic stress disorder symptoms and psychosocial functioning in Veterans of Operations Enduring Freedom and Iraqi Freedom. Psychiatry Research, 178, 323-329.

Raftery, A. E. (1995). Bayesian model selection in social research. Sociological Methodology, 25, 111-163.

Shevlin, M., McBride, O., Armour, C., \& Adamson, G. (2009). Reconciling the differences between the King et al. (1998) and Simms et al. (2002) factor models of PTSD. Journal of Anxiety Disorders, 23, 995-1001.

Simms, L. J., Watson, D., \& Doebbeling, B. N. (2002). Confirmatory factor analyses of posttraumatic stress symptoms in deployed and nondeployed veterans of the Gulf War. Journal of Abnormal Psychology, 111, 637-647.

Spielberger, C. D., Gorsuch, R. L., Lushene, R., Vagg, P. R., \& Jacobs, G. A. (1983). Manual for the state-trait anxiety inventory (Form Y). Palo Alto, CA: Consulting Psychologists Press.

Vigneau, F., \& Cormier, S. (2008). The factor structure of the state-trait anxiety inventory: An alternative view. Journal of Personality Assessment, 90, 280-285.

Watson, D. (2005). Rethinking the mood and anxiety disorders: A quantitative hierarchical model for DSM-V. Journal of Abnormal Psychology, 114, 522-536.

Wang, L., Li, Z., Shi, Z., Zhang, J., Zhang, K., Liu, Z., et al. Testing the dimensionality of posttraumatic stress responses in young Chinese adult earthquake survivors: Further evidence for 'dysphoric arousal' as a unique PTSD construct. Depression and Anxiety, in press. 
Wang, L., Long, D., Li, Z., \& Armour, C. (2011). Posttraumatic stress disorder symptom structure in Chinese adolescents exposed to a deadly earthquake. Journal of Abnormal Child Psychology, 39, 749-758.

Wang, L., Zhang, J., Shi, Z., Zhou, M., Li, Z., Zhang, K., et al. (2011). Comparing alternative factor models of PTSD symptoms across earthquake victims and violent riot witnesses in China: Evidence for a five-factor model proposed by Elhai et al. (2011). Journal of Anxiety Disorders, 25, 771-776.

Weathers, F. W., Litz, B. T., Herman, D. S., Huska, J. A., \& Keane, T. M. (1993). The PTSD checklist: Reliability, validity, \& diagnostic utility. In Paper presented at the annual meeting of the International Society for Traumatic Stress Studies San Antonio, TX.
Wetherell, J. L., \& Areán, P. A. (1997). Psychometric evaluation of the Beck Anxiety Inventory with older medical patients. Psychological Assessment, 9, 136-144.

Wirth, R. J., \& Edwards, M. C. (2007). Item factor analysis: Current approaches and future directions. Psychological Methods, 12, 58-79.

Yu, C. Y. (2002). Evaluating cutoff criteria of model fit indices for latent variable models with binary and continuous outcomes. Doctoral dissertation, University of California, Los Angeles.

Yufik, T., \& Simms, L. J. (2010). A meta-analytic investigation of the structure of posttraumatic stress disorder symptoms. Journal of Abnormal Psychology, 119, 764-776. 